\title{
Pollen Tube Growth and Double Fertilization in Camellia oleifera
}

\author{
Chao Gao, Deyi Yuan', Ya Yang, Bifang Wang, Dongming Liu, and Feng Zou \\ Key Laboratory of Cultivation and Protection for Non-Wood Forest Trees of Ministry of Education \\ and the Key Laboratory of Non-Wood Forest Products of Forestry Ministry, Central South University \\ of Forestry and Technology, Changsha 410004, China
}

\begin{abstract}
AdDitional INDEX wORDS. crossbreeding, fluorescence microscopy, hybridization, reproduction, scanning microscopy, angiosperms, plant

Abstract. Camellia oleifera is an important plant species that produces edible oils. Understanding the double fertilization of this plant is critical for studies concerning crossbreeding, self-incompatibility, and the biological mechanisms underlying hybridization. We aimed to characterize pollen tube growth and double fertilization in C. oleifera. The female and male parent cultivars (Huashuo and Xianglin XLC15, respectively) were used for artificial pollination. Growth of the pollen tube in the style, ovary, and ovule from pollination to fertilization and the cytological characteristics of female and male gamete fusion during double fertilization were observed using fluorescence and scanning electron microscopy (SEM). Numerous pollen grains germinated 2 to 4 hours after pollination. The pollen tubes entered the interspaces between the papillar cells, grew along the stylar canal, and aggregated at the one-third site of the style. They grew in the gradually narrowing stylar canal, entering the locule. The tubes turned $90^{\circ}$ and entered the embryo sac through the micropyle; subsequently, they entered a degenerated synergid, where the spermatids were released. One sperm nucleus fused with the polar nucleus, forming the primary endosperm nucleus, whereas the other sperm fused with the egg, forming the zygote. The polar nucleus was fertilized earlier than the egg. Double fertilization of $C$. oleifera is characterized as pre-mitotic gametogony. The current results lay a theoretical foundation for studies concerning the crossbreeding and embryology of $C$. oleifera and provide fundamental data concerning the reproductive biology of the genus Camellia.
\end{abstract}

The compatible fertilization of angiosperms is initiated through the hydration of the pollen at the stigma and the germination of the pollen tube. After entering the interpapillar cell space, the tube grows in the stylar canal or transmitting tissue until it enters the ovary. Subsequently, the tube penetrates the embryo sac through the micropyle and enters degenerated synergids, where it releases two spermatids: one sperm fuses with an egg to form a fertilized egg and the other sperm fuses with the central cell to form the endosperm, thereby fulfilling the process of double fertilization. Biological fertilization events from pollen germination to gamete fusion are complex and delicate with strict timing and spatiality; the underlying laws and characteristics of these processes vary depending on the plant (Ge et al., 2007; Weterings and Russell, 2004). For example, based on studies of double fertilization in Oryza sativa (Ding et al., 2009), the egg and the sperm fuse to form a zygote at 0.5 to $2.5 \mathrm{~h}$ after pollination. At $10.0 \mathrm{~h}$, the zygote is split into the two-celled proembryo for the first time. Zygophase is referred to as a period of pollination that lasts from 2.5 to $10.0 \mathrm{~h}$. Because they are pollinated for 1.0 to $3.0 \mathrm{~h}$, the sperm nucleus and the two polar nuclei fuse. After being pollinated for $5.0 \mathrm{~h}$, the primary endosperm nuclei began to split. However, in Brassica campestris ssp. pekinensis, zygotes were formed after being pollinated for $24 \mathrm{~h}$, and they split for the first time after

Received for publication 6 June 2014. Accepted for publication 7 Oct. 2014. This work was supported by the National Natural Science Foundation of China (31170639), the Graduate Scientific Research Foundation of Central South University of Forestry and Technology (CX2013A01), and the Hunan Provincial Innovation Foundation of Postgraduates (CX2013A014).

We thank Prof. Huiqiao Tian for performing the reproductive biology analyses and Dr. Ting Liao for assistance with the field work.

${ }^{1}$ Corresponding author. E-mail: yuan-deyi@163.com. being pollinated for 32 to $34 \mathrm{~h}$. After being pollinated for $22 \mathrm{~h}$, primary endosperm nuclei were formed. After being pollinated for $24 \mathrm{~h}$, mitosis was observed for the first time (Peng and Shen, 2005). Fertilization is an important biological event in plant sexual reproduction and has become an important topic in studies on plant embryology. Since the first report describing double fertilization, great progress has been made in many studies. For the study of male and female germ units, the model plant Arabidopsis thaliana has been selected as the main experimental model. Studies on the segregation and function of male and female germ units have enriched theories of angiosperm reproductive biology (Ge et al., 2011; Huang and Russell, 1992). To elucidate the interactions between pollen and stigma, Ma et al. (2013) comprehensively analyzed studies examining the characteristics of Corylus plants after selfcrossing or outcrossing pollination, focusing on the affinity between pollen and stigma. Their research has laid a theoretical foundation for improving fruit quality and understanding the evolution of Corylus plants. Higashiyama et al. (2000) examined the events occurring after pollen tubes entered the embryo, describing the detailed process of spermatid release inside degenerated synergids after the pollen tubes of Torenia fournieri entered the embryo. Berger et al. (2008) performed studies on spermatids and ova, comprehensively exploring their interrecognition and fusion with each other. These studies provided vital information to allow control of plant fertilization to increase agricultural output and reproduction.

Camellia oleifera is an evergreen shrub or small tree belonging to the genus Camellia, a flowering plant in the family Theaceae (Fig. 1A and B), and it is an important woody tree plant producing edible oil in southern China. Camellia has a wide range of uses with a long cultivation history. C. oleifera 


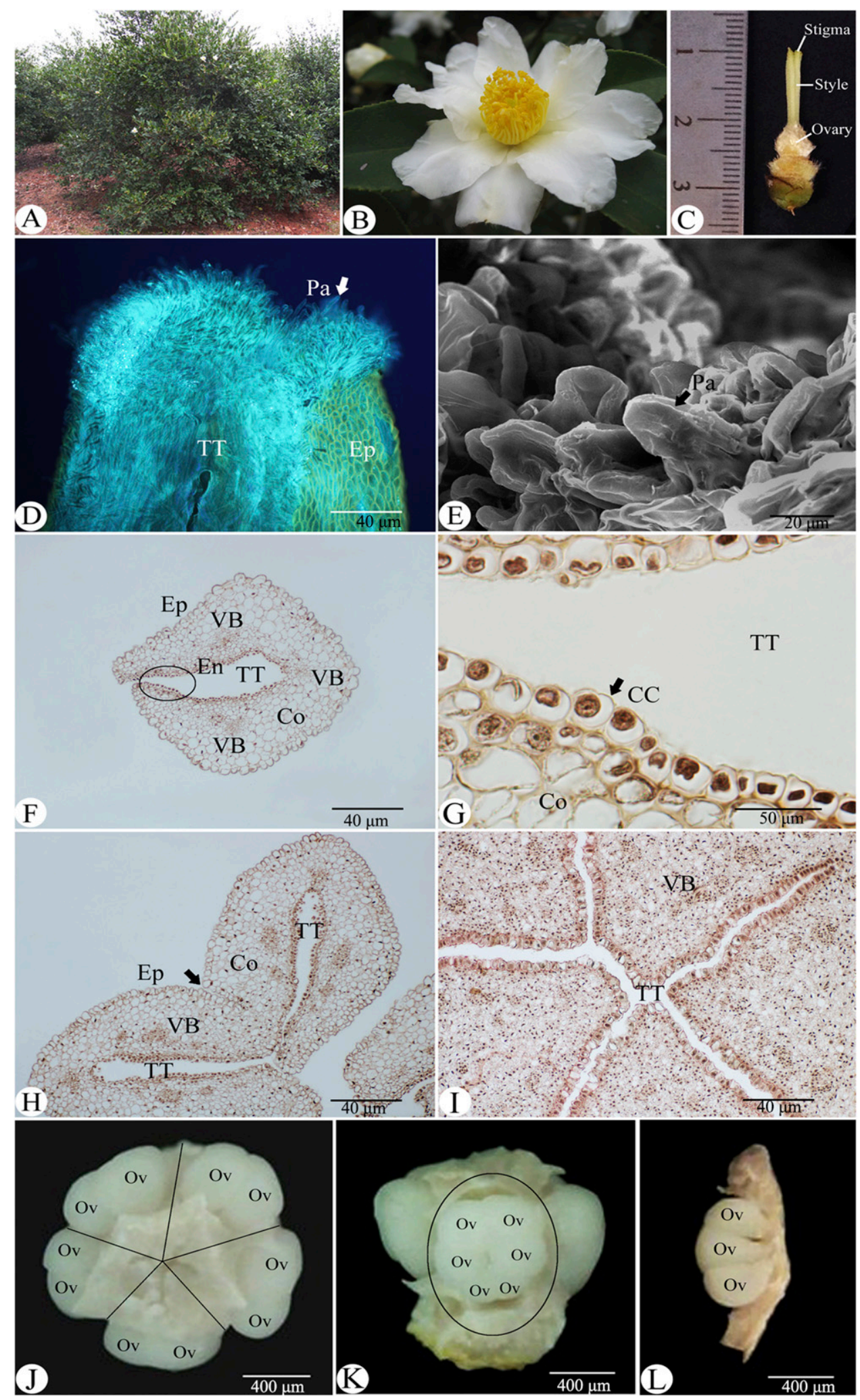

Fig. 1. The tree body, floral organs, and pistil of Camellia oleifera: (A) the adult tree body, (B) the floral organ, (C) the pistil. (D) The papillar cells were densely distributed on the stigma $(\times 100)$, and the surface that could not accept pollen comprised the epidermis (Ep). (E) Papillar cells were observed on the surface of the stigma. (F) The transection of the style shows three vascular bundles $(\times 100)$. (G) A partially enlarged view of the area within the red circle in $\mathbf{F}$, the stylar canal, showing transmitting cells with regular morphology and a large nucleus $(\times 100)$. (H) The styles began to attach from the two-thirds site downward $(\times 100)$. (I) The stylar canals converged to form an interconnected structure in the ovary, and the transmitting tissue cells in the innermost layer can be observed $(\times 100)$. (J) Polar view (top) of the ovary: five locules are divided with straight lines $(\times 25)$. (K) Equatorial view of the ovary: the ellipse shows one locule with two columns of ovules $(\times 25)$. (L) One column of ovules inside a seed bud $(\times 25)$; bars: $\mathbf{E}=20 \mu \mathrm{m} ; \mathbf{D}, \mathbf{F}, \mathbf{H}, \mathbf{I}=40 \mu \mathrm{m} ; \mathrm{G}=50 \mu \mathrm{m} ; \mathbf{J}, \mathbf{K}, \mathbf{L}=400 \mu \mathrm{m}$. CC $=$ canal cell; $\mathrm{Co}=$ cortex; $\mathrm{En}=$ endodermis; $\mathrm{Ep}=$ epidermis; $\mathrm{Ov}=$ ovule; $\mathrm{Pa}=$ papilla; $\mathrm{PT}=$ pollen tube; $\mathrm{TT}=$ transmitting tissue; $\mathrm{VB}=$ vascular bundle. 
is mainly cultivated for its seed, and the manufacture of edible oils from Camellia seed is the primary cultivation objective; the edible oil obtained from $C$. oleifera is referred to as "eastern olive oil" as a result of its high quality (Lee and Yen, 2006). Although researchers have conducted numerous studies on the double fertilization of model plants and crops (Ding et al., 2009; Faure et al., 2002; Li et al., 2009; Mol et al., 1994), studies on the sexual reproduction of woody plants are rare, particularly those of the genus Camellia. Furthermore, even studies on the sexual fertilization of the genus Camellia have primarily focused on the development of the anther, ovules, megaspores, microspores, female gametocytes, and male gametocytes (Kapil and Sethi, 1963; Tsou, 1997; Zou et al., 2013a, 2013b); self-incompatibility (Chen et al., 2012; Wachira and Kamunya, 2005); and seed growth and development (Mondal, 2011; Sethi, 1965). Currently, there are no reports concerning fertilization, which is essential for the sexual reproduction of $C$. oleifera and is closely associated with the breeding of this plant.

Therefore, in this study, we explored the processes of pollen tube growth and fertilization to more clearly understand sexual reproduction of $C$. oleifera after pollination. Fluorescence microscopy and SEM were used to observe the development of pollinated pollen tubes of $C$. oleifera in the stigma, inside the style, and on entering the seed bud and micropyle. Paraffin sections were selected to observe the release of spermatids after pollen tubes entered the embryo and the formation of a fertilized egg cell and endosperm after the spermatids, respectively, fused with the polar nucleus and the egg cell. To some extent, the current study has laid a theoretical foundation and provided useful data for crossbreeding and embryological studies in $C$. oleifera.

\section{Materials and Methods}

Materials. The cultivar Huashuo was clonally propagated through grafting and cultivated at the research workstation at Central South University of Forestry and Technology in Changsha, China (lat. $28^{\circ} 05^{\prime} \mathrm{N}$, long. $113^{\circ} 21^{\prime} \mathrm{E}$ ). Before the experiment, the plants were grown for 5 years and exhibited normal blossoming and fruit-bearing capacities. At this work station, the annual mean amount of precipitation is $1380 \mathrm{~mm}$ and the annual mean temperature is $19.3{ }^{\circ} \mathrm{C}$ with an annual $10^{\circ} \mathrm{C}$ or greater accumulated temperature of $5463^{\circ} \mathrm{C}$, an annual mean frost-free duration of 276 to $291 \mathrm{~d}$, and an annual mean sunshine duration of $1762 \mathrm{~h}$. The conditions during the experimental period, including climate and rainfall conditions, were basically consistent with the annual mean conditions. This area also has a humid subtropical monsoon climate with sufficient sunshine and a short winter. The plants were cultivated with acid red soil, which is typical in subtropical areas with low hills. The plants were watered weekly with $15 \mathrm{~L}$ of water provided to each plant. Plants in good health that were able to typically bloom and bear fruit were selected from the same lot $\left(\approx 500 \mathrm{~m}^{2}\right)$, and samples of flower buds that were near bloom at the full flowering state were collected at $0900 \mathrm{HR}$. The anthers were emasculated before powder loosening, and artificial pollination was performed. The cultivar used in this study was Huashuo, a nationally certified, large-fruited Camellia cultivar (Tan et al., 2011) that is cultivated at the Central South University of Forestry and Technology, Changsha, China (CSUFT). The pollinating $C$. oleifera cultivar was Xianglin XLC15 (Wang et al., 2008), which was provided by CSUFT.
FLUORESCENCE MICROSCOPY AND PARAFFIN SECTIONING. The pistils were collected before pollination and at $1,2,4,12,36$, $48,54,60,66,72,78,84,90,96,108,120,144$, and $168 \mathrm{~h}$ after pollination. The pistil samples were fixed in Carnoy's solution (at an acetic acid:ethanol ratio of 1:3) for $5 \mathrm{~h}$ and subsequently maintained in 70\% alcohol (Ding et al., 2009). The pistils were softened for $5 \mathrm{~h}$ in $8 \mathrm{~mol} \cdot \mathrm{L}^{-1} \mathrm{NaOH}$ before observation. Then, the pistils were washed with distilled water until no $\mathrm{NaOH}$ remained. The softened style was torn open along the stigma and stained for $5 \mathrm{~h}$ with $0.5 \%$ water-soluble aniline blue (prepared in $0.15 \mathrm{~N}$ dipotassium hydrogen phosphate buffer). Routine pallet pressing was performed. The growth of the pollen tube in the style was observed (Kho and Baer, 1968) under a fluorescence microscope (BX-51; Olympus, Tokyo, Japan), and images were captured.

The double fertilization process was observed using a conventional paraffin sectioning method, which is a modified version of the method described by Kapil and Sethi (1963). Hematoxylin staining and iron alum-hematoxylin section staining were performed; the section thickness was $8 \mu \mathrm{m}$. The sections were observed under a fluorescence microscope (BX-51).

Pollen tube Growth Was observed using SEM. The pollen tubes were prefixed in $2.5 \%$ glutaraldehyde solution (prepared with $0.1 \mathrm{~mol} \cdot \mathrm{L}^{-1}$ phosphate buffer) for $2 \mathrm{~h}$. After washing three times (30 min each wash), the samples were fixed in 1\% osmic acid solution for $2 \mathrm{~h}$ followed by additional washing and dehydration using a gradient ethanol series. The pollen tubes were transferred to tertiary butyl alcohol followed by cryodessication. The samples were placed in an ion sputtercoater and gilded for $20 \mathrm{~min}$. The pollen tubes were observed using a SEM (JSM-6390; JEOL, Tokyo, Japan), and images were obtained.

\section{Results}

STyle AND OVARIES. The pistil of $C$. oleifera comprises the stigma, style, and ovary (Fig. 1C). The stigma was wet, comprising acceptor and non-acceptor surfaces. Tiny papillar cells were densely distributed on the acceptor surface. This surface acted as a pollen "catcher," which maximally extended during blooming to accept as many pollen grains as possible (Fig. 1D and E). The styles were $\approx 12 \mathrm{~mm}$ in length, and the number of the styles ranged from four to five. Typically, the upper two-thirds region of the style was split apart (Fig. 1F), and the lower one-third region was interconnected (Fig. 1H). The style was hollow (Fig. 1F), and the inner surface was composed of the outer epidermis, cortex, and endodermis. The endodermis was the pathway for the growth of the pollen tube (Fig. 2C). Epidermal cells secreted mucosubstance on the surface of the stylar canal. The canal cell layer was thicker than the layer of parenchymatous tissue and served as a path for the pollen tubes. The cells had a normal morphology with a large nucleus and the characteristics of glandular cells (Fig. $1 G)$. The stylar canals showed a gradual trend of attachment from top to bottom: the lower the site, the denser the attachment and the smaller the interspace. Particularly, the stylar canals independently attached to each other from the base of the styles to the ovaries, forming a narrow crevice (Fig. 1I). In C. oleifera, the number of locules was equivalent to the number of styles. The ovary typically comprised four to five chambers formed by carpels and was $\approx 5 \mathrm{~mm}$ in length with axile placentation. In each chamber, two lines of anatropous ovules grew with two to 


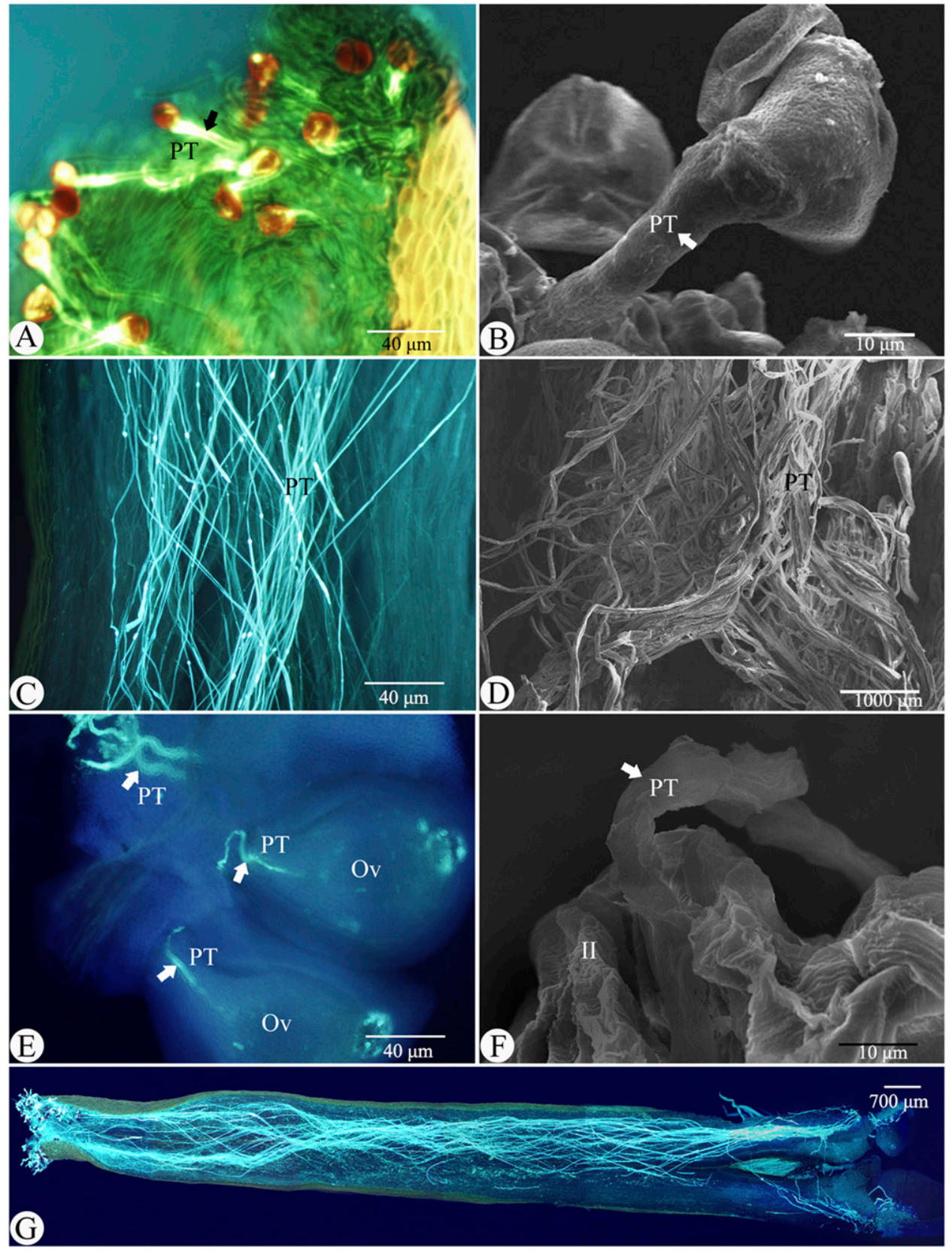

Fig. 2. The growth of the stylar tube of Camellia oleifera. (A) Pollen grains germinated from the stigma and, subsequently, pollen tubes grew into the interspaces between the papillar cells (fluorescence micrograph $\times 200$ ). (B) A pollen tube grew out of the germ pore of a pollen grain and subsequently entered the papillar cells (scanning electron micrograph). (C) The pollen tubes grew downward into the style in a staggered manner, and callose plugs can be observed (fluorescence micrograph $\times 200$ ). (D) Thin and flat pollen tubes grew into the stylar canal in a staggered manner (scanning micrograph). (E) The pollen tube entered the embryo sac along the placenta through the funiculus after entering the ovary (fluorescence micrograph $\times 100)$. $(\mathbf{F})$ The pollen tube made a $90^{\circ}$ turn at the micropyle (scanning micrograph). (G) A complete view of pollen tube growth in the style and ovary: the tubes aggregated at the one-third site of the style $(\times 40)$ and became denser after reaching the base of the style; bars: $\mathbf{A}, \mathbf{C}, \mathbf{E}=40 \mu \mathrm{m} ; \mathbf{B}, \mathbf{F}=10 \mu \mathrm{m} ; \mathbf{D}=1000 \mu \mathrm{m} ; \mathbf{G}=700 \mu \mathrm{m}$. Ov $=$ ovule; PT = pollen tube; II = inner integument

three ovules in each line (Fig. 1J-L). One side of the ovules in C. oleifera grew faster than the other side. The side that grew more slowly was inverted $\approx 180^{\circ}$, but the nucellus was not bent. The micropyle was set at one side of the ovule stalk, and the convergence point was often set at its opposite side. The external integument near one side of the ovule stalk was often adnated into one single-band raphe. Consequently, the attachment line of convergence, the nucellus, and the micropyle were nearly parallel with the ovule stalk, forming anatropous ovules. The ovules were also tenuinucellate with a double integument.

Pollen germination AND POLlen TUBE GROWTH IN THE STYLE AND OVARY. At 2 to $4 \mathrm{~h}$ after pollination, the pollen tubes germinated and grew out; they entered the stigma, whereas the pollen grains remained outside (Fig. 2A and B). After the pollen tubes entered the stigma, they converged inside the style (Fig. 2C, D, and G). At $40 \mathrm{~h}$, the pollen tubes reached the base of the style and subsequently entered the ovule (Fig. 2E and G). When the pollen tube approached the micropyle of the ovule, it made a $90^{\circ}$ turn to enter the micropyle and later the embryo sac (Fig. 2E and F).

DOuBLE FERTILIZATION. At blooming, the mature embryo sac had a typical seven-cell and eight-nucleus structure [Fig. 3A-3D; Fig. 3A, two synergids; Fig. 3B, two polar nuclei (central cells); Fig. 3C, an egg cell; Fig. 3D, three antipodal cells (nuclei)]. An egg apparatus, comprising two synergids (Fig. 3A) and an egg cell (Fig. 3C) was located at the micropylar end. The synergid had a pear shape with noticeable polarity. There was a large vacuole at each chalazal end of the synergid. The nucleus of the egg cell was typically located near the chalazal end; however, it was also occasionally located in the middle of the cell. A vacuole was located at the micropylar end of the cell. The central cell was located in the middle of the embryo sac near two close but non-fused polar nuclei (sometimes, these two nuclei were fused to form a secondary nucleus) (Fig. 3B). The polar nuclei were typically located below or around the egg apparatus; both nuclei were also occasionally located in the middle of the central cell. Before fertilization, degenerated antipodal cells could be observed at the chalazal end of the embryo sac, although some embryo sacs lacked these cells (Fig. 3D). As the serial sections show, traces of pollen tubes that had entered the micropyle were left behind (Fig. 3E), and two spermatids appeared inside one degenerated synergid. The nuclei of these spermatids were deeply colored, although the male nucleolus could not be distinguished unambiguously, because it was not completely separated and exhibited an ellipsoid shape (Fig. 3F). Serial sections showed that 


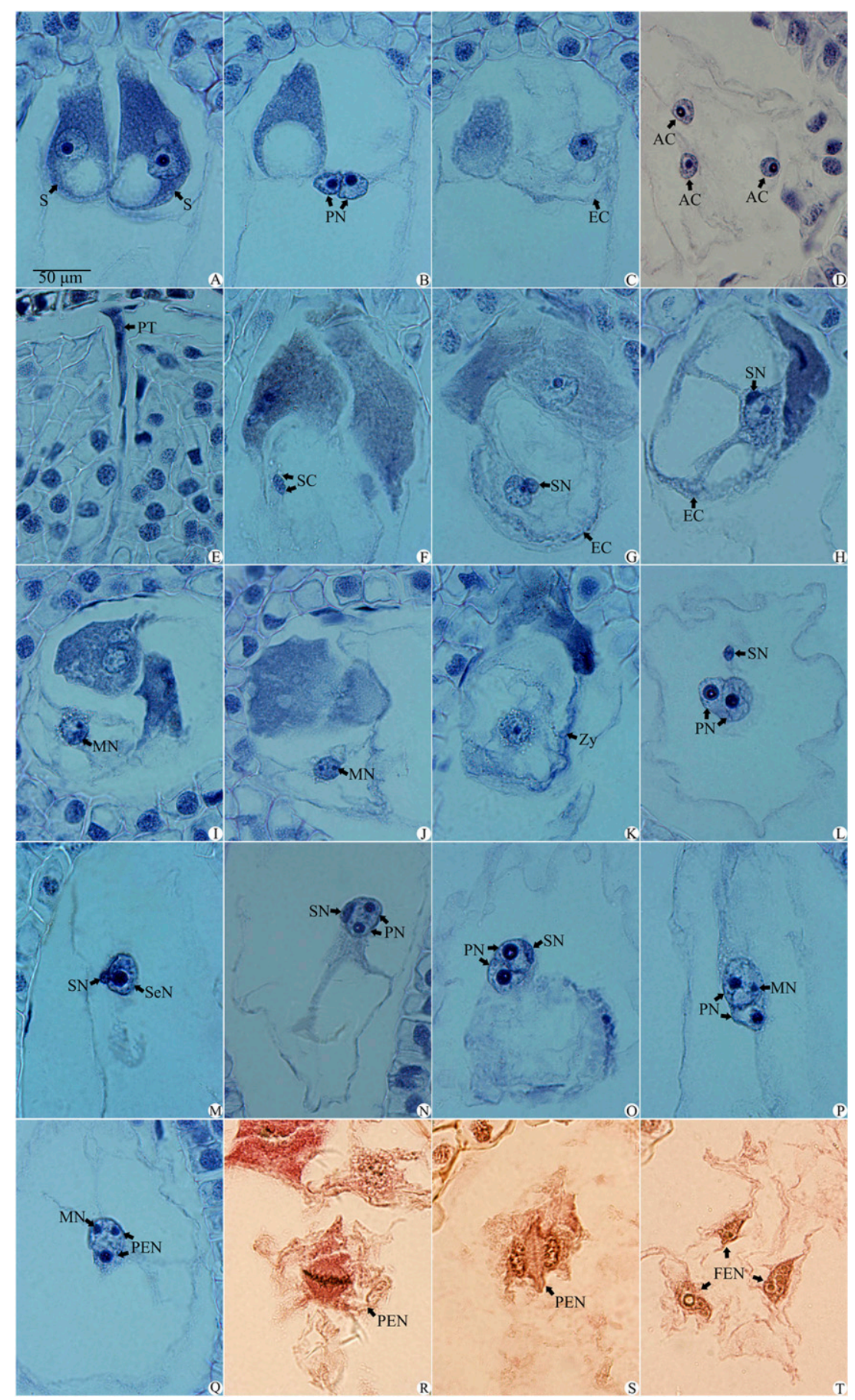

Fig. 3. The egg apparatus of the mature embryo sac and the process of double fertilization of Camellia oleifera. (A) Two pear-shaped synergids (indicated by arrows) with noticeable polarity can be observed at the micropylar end $(\times 1000)$. (B) During embryo sac maturation, the polar nuclei (indicated by arrows) were located near the egg apparatus $(\times 1000)$. (C) The polarity was indistinguishable when the nucleus was located in the middle of the egg cell (indicated by an arrow) $(\times 1000)$. (D) During embryo sac maturation, antipodal cells (indicated by arrows) deteriorated $(\times 1000)$. (E) The pollen tube (indicated by an arrow) entered the embryo sac through the micropyle, and the micropyle was fertilized $(\times 1000)$. (F) The pollen tube entered a synergid and released two sperm cells (indicated by arrows); the cells were not separated from each other $(\times 1000)$. (G) The sperm nucleus (indicated by an arrow) approached the egg cell (indicated by an arrow) $(\times 1000)$. (H) The sperm nucleus (indicated by an arrow) attached to the egg nucleus (indicated by an arrow) and gradually fused $(\times 1000)$. (I) Male chromatin dispersed into the egg nucleus $(\times 1000)$. (J) A male nucleus (indicated by an arrow) appeared in the egg nucleus $(\times 1000)$. (K) The zygote (indicated by an arrow) $(\times 1000)$. (L) The sperm nucleus (indicated by an arrow) migrated toward the polar nucleus, and at this time, the polar nucleus (indicated by an arrow) was located far from the egg apparatus $(\times 1000)$. (M) The sperm nucleus (indicated by an arrow) approached the secondary nucleus (indicated by an arrow) $(\times 1000)$. (N) The sperm nucleus (indicated by an arrow) attached to the polar nucleus (indicated by arrows) and gradually fused $(\times 1000)$. (O) Male chromatin dispersed into the polar nucleus (indicated by an arrow) $(\times 1000)$. (P) A male nucleus (indicated by an arrow) appeared in the polar nucleus (indicated by an arrow) $(\times 1000)$. (Q) A primary endosperm nucleus (indicated by an arrow) formed; the three nuclei were not fused at this time $(\times 1000)$. (R) The primary endosperm nucleus (indicated by an arrow) split at metaphase $(\times 1000)$. (S) The primary endosperm nuclei (indicated by an arrow) continued to split $(\times 1000)$. (T) Free endosperm nuclei (indicated by arrows $)$ in the embryo sac $(\times 1000)$; bars: $\mathbf{A}-\mathbf{T}=50 \mu \mathrm{m}$. AC $=$ antipodal cell; $\mathrm{EC}=$ egg cell; $\mathrm{FEN}=$ free endosperm nucleus; $\mathrm{MN}=$ male nucleus; $\mathrm{PEN}=$ primary endosperm nucleus; $\mathrm{PN}=$ polar nucleus; $\mathrm{PT}=$ pollen tube; $\mathrm{S}=$ synergid; $\mathrm{SeN}=$ secondary nucleus; $\mathrm{SC}=$ sperm cell; $\mathrm{SN}=$ sperm nucleus; $\mathrm{Zy}=$ zygote. 
the male nucleus moved toward the polar nucleus before moving toward the egg. The male nucleus attached to the membrane of the egg nucleus (Fig. 3G), and the membranes of the male and female nuclei fused (Fig. 3H). The male chromatin diffused (Fig. 3I), and a small male nucleolus appeared (Fig. 3J). The male and female nuclei were blended into a zygote (Fig. 3K) at 66 to $144 \mathrm{~h}$ after pollination. The male nucleus moved toward the polar nucleus inside the central cell (Fig. 3L). It adhered to the membrane of the polar nucleus, and the male nucleus resembled a convex lens at this moment (Fig. 3M). The membranes of the male and polar nuclei fused (Fig. $3 \mathrm{~N}$ ). Dispersed male chromatin can be observed inside the polar nucleus (Fig. 3O). Subsequently, a male nucleus appeared (Fig. 3P). This nucleus grew, and then the female nuclei fused to form the primary endosperm nucleus (Fig. 3Q) at 54 to $120 \mathrm{~h}$ after pollination. After the polar nucleus (secondary nucleus) was fertilized, the primary endosperm nucleus split to form a few free endosperm nuclei inside the embryo sac. The endosperm of $C$. oleifera was extremely undeveloped (Fig. 3R-T).

\section{Discussion}

Pollination and fertilization result from interactions between compatible pollen grains and pistils (Aliyu, 2007). In $C$. oleifera, we observed that almost all pollen grains on the stigma germinated and successfully grew in the style, which completed the first step of affinity pollination (Ram et al., 2008). As the styles transitioned toward the ovary, the stylar canals became denser, and the pollen tubes assembled at the one-third site of the pollen tubes. According to $\mathrm{Hu}$ (2005), the fertilization of angiosperms exhibits porogamy, misogamy, and chalazogamy. In $C$. oleifera, the ovule contains two layers of integument, and the micropyle is formed from the inner integument. The pollen tube enters the locule with the aid of pollen tube transmitting tissue, turns $90^{\circ}$ along the funiculus, and enters the micropyle. Therefore, the fertilization pattern of C. oleifera is porogamy, which is the same as that of Chrysanthemum grandiflorum (Deng et al., 2010) and Iris mandshurica. (Zhang et al., 2011). The entire fertilization process of $C$. oleifera was consistent with that of porogamy plants, which was reported by Ge et al. (2007). Although the edible oil obtained from $C$. oleifera is known as "eastern olive oil," the characteristics of the pollination and fertilization of C. oleifera are quite different from those of Olea europaea, which is another important woody tree plant that produces edible oil. The latter species has a stigma that is shorter than that of $C$. oleifera. Similarly, the pollen tubes of $O$. europaea grow between cells of the transmitting tissue of the style, but only one pollen tube enters the ovary and then the embryo sac through the micropyle. In O. europaea, zygotes are observed at $9 \mathrm{~d}$ after blooming, and suspensors develop at 4 to 5 weeks. At $5 \mathrm{~d}$ after pollination, the endosperm nucleus begins to develop, and at $15 \mathrm{~d}$ after pollination, cellularization occurs. Although there are two ovules in each ventricle of $O$. europaea, only one ovule develops into a mature seed. During embryo development, the nucellus and endosperm are completely absorbed (Ateyyeh et al., 2000; Reale et al., 2006). These reproductive characteristics of $O$. europaea are in contrast with the long-time dormancy of the zygote and the extremely undeveloped endosperm in C. oleifera.

Furthermore, in $C$. oleifera, we observed that the plasma membranes that are in close proximity of each other fuse at one site first during the fusion of the female and male gametes followed by multisite fusion. The fused membrane gradually disintegrates until the cytoplasm and nucleus of the sperm enter the female cell. During this process, the plasma membrane of the spermatid participates in the construction of the plasma membrane of the zygote. Russell et al. assumed that angiosperms have five gametogony patterns (Russell, 1992; Russell et al., 1990), although some scholars have suggested that the five patterns can be divided into two categories (Ding et al., 2009; Yu et al., 1994). The results of the present study suggest that sperm cytoplasm participates in the gametogony of C. oleifera. The same phenomenon was also found in Nicotiana tabacum (Yu et al., 1994) and Plumbago zeylanica (Russell, 1992). However, several issues remain. For example, the detailed process of plasma membrane fusion between the spermatid and egg remains unknown, and there is a lack of evidence based on ultrastructural studies to support the observations in the present study. In addition, according to Weterings and Russell (2004), the spermatids, polar nuclei, and egg change locations after fertilization and double fertilization during the movement of the embryo sac, which is an interesting phenomenon. In the present study, we observed that the embryo sac of $C$. oleifera matured during blooming, and the polar nuclei were located near the egg apparatus. As pollination and fertilization proceeded, particularly after the release of the pollen tube contents and the two spermatids, the location of the polar nuclei changed during the fusion of the spermatids with the egg and polar nuclei. Specifically, the polar nuclei gradually moved away from the egg apparatus and were freed in the middle or the margin of the embryo sac to fuse with the spermatid. However, when the spermatid fused with the egg, a change in the position of the egg was not observed, and this cell remained near the micropylar end. Thus, the mechanism underlying the fusion that occurs after the polar nuclei change locations requires further exploration. Nevertheless, the following phenomenon was revealed in the present study: the fusion between the sperm and polar nuclei occurred earlier than the fusion between the sperm and egg. The same phenomenon was also observed in O. sativa (Ding et al., 2009) and Sorghum bicolor (Li et al., 2009).

The smooth progression of sexual reproduction of higher plants depends on the healthy development of female and male gametocytes. The precise fusion of male and female gametes and the formation of a zygote during double fertilization are affected through the self-development of the plant and the surrounding environment, including the species characteristics of the plant, nutritional status of the plant, ambient temperature, illumination, and water supply, among other factors (Kakani et al., 2005; Snider et al., 2011). The flowering season of C. oleifera lasts from the end of October until the last third of December every year in the northern hemisphere with the fullbloom stage occurring in the middle of November (Tan et al., 2011). As a species that blooms in fall, the pollination and fertilization of $C$. oleifera often accompanies low temperatures and cloudy, rainy weather conditions. In the present study, the growth of the $C$. oleifera pollen tube, from germination to the base of the style, required $\approx 40 \mathrm{~h}$. This duration is longer than that of $O$. sativa (Ding et al., 2009) and B. campestris ssp. pekinensis (Peng and Shen, 2005). The question of whether the botanical characteristics of $C$. oleifera or the low temperature during fertilization determines the duration of pollen tube growth is worth further exploration. 


\section{Literature Cited}

Aliyu, O.M. 2007. Pollen-style compatibility in cashew (Anacardium occidentale L.). Euphytica 158:249-260.

Ateyyeh, A.F., R. Stosser, and M. Qrunfleh. 2000. Reproductive biology of the olive (Olea europaea L.) cultivar 'Nabali Baladi'. J. Appl. Bot. Food Quality 74:255-270.

Berger, F., Y. Hamamura, M. Ingouff, and T. Higashiyama. 2008. Double fertilization-Caught in the act. Trends Plant Sci. 13:437443.

Chen, X., S. Hao, L. Wang, W. Fang, Y. Wang, and X. Li. 2012. Lateacting self-incompatibility in tea plant (Camellia sinensis). Biologia 67:347-351.

Deng, Y., N. Teng, S. Chen, F. Chen, Z. Guan, A. Song, and Q. Chang. 2010. Reproductive barriers in the intergeneric hybridization between Chrysanthemum grandiflorum (Ramat.) Kitam. and Ajania przewalskii Poljak. (Asteraceae). Euphytica 174:41-50.

Ding, J.T., J.H. Shen, W. Li, and H. Yang. 2009. Cytological observation of double fertilization and its duration in Oryza sativa. Chinese Bul. Bot. 44:473-483 [in Chinese with English abstract].

Faure, J.E., N. Rotman, P. Fortune, and C. Dumas. 2002. Fertilization in Arabidopsis thaliana wild type: Developmental stages and time course. Plant J. 30:481-488.

Ge, L.L., X.P. Gou, T. Yuan, G.W. Strout, J. Nakashima, E.B. Blancaflor, H.Q. Tian, and S.D. Russell. 2011. Migration of sperm cells during pollen tube elongation in Arabidopsis thaliana: Behavior during transport, maturation and upon dissociation of male germ unit associations. Planta 233:325-332.

Ge, L.L., H.Q. Tian, and S.D. Russell. 2007. Calcium function and distribution during fertilization in angiosperms. Amer. J. Bot. 94:1046-1060.

Higashiyama, T., H. Kuroiwa, S. Kawano, and T. Kuroiwa. 2000. Explosive discharge of pollen tube contents in Torenia fournieri. Plant Physiol. 122:11-14.

Hu, S.Y. 2005. Reproductive biology of angiosperms. China Higher Educ. Press, Beijing, China.

Huang, B.Q. and S.D. Russell. 1992. Female germ unit: Organization, isolation, and function. Intl. Rev. Cytol. 140:233-293.

Kakani, V.G., K.R. Reddy, S. Koti, T.P. Wallace, P.V.V. Prasad, V.R. Reddy, and D. Zhao. 2005. Differences in in vitro pollen germination and pollen tube growth of cotton cultivars in response to high temperature. Ann. Bot. (Lond.) 96:59-67.

Kapil, R.N. and S.B. Sethi. 1963. Development of male and female gametophytes in Camellia sinensis (L.) O. Kuntze. Proc. Natl. Inst. Sci. India B 29:574-597.

Kho, Y.O. and J. Baer. 1968. Observing pollen tubes by means of fluorescence. Euphytica 17:298-302.

Lee, C.P. and G.C. Yen. 2006. Antioxidant activity and bioactive compounds of tea seed (Camellia oleifera Abel.) oil. J. Agr. Food Chem. 54:779-784.

Li, R.L., J.H. Shen, Y. Jia, W. Li, and L.M. Wang. 2009. Fertilization process in sorghum and its performance time for each stage. Acta Agron. Sin. 35:2234-2242 [in Chinese with English abstract].

Ma, Q.H., G.X. Wang, W.J. Liang, X. Chen, L.S. Liang, and T.T. Zhao. 2013. Progress on pollen-stigma compatibility in Corylus (hazelnuts): A review. J. For. Res. 24:397-402.
Mol, R., E. Matthys-Rochon, and C. Dumas. 1994. The kinetics of cytological events during double fertilization in Zea mays L. Plant J. 5:197-206.

Mondal, T.K. 2011. Camellia, p. 15-39. In: Kole, C. (ed.). Wild crop relatives: Genomic and breeding resources. Springer, Berlin/ Heidelberg, Germany.

Peng, J. and J.H. Shen. 2005. On the process of fertilization in Brassica campestris ssp. pekinensis and its duration of each stage. Acta Hort. Sinica 32:812-817 [in Chinese with English abstract].

Ram, S.G., V. Thiruvengadam, S.H. Ramakrishnan, and J.R.K. Bapu. 2008. Investigation on pre-zygotic barriers in the interspecific crosses involving Gossypium barbadenseand and four diploid wild species. Euphytica 159:241-248.

Reale, L., C. Sgromo, T. Bonofiglio, F. Orlandi, M. Fornaciari, F. Ferranti, and B. Romano. 2006. Reproductive biology of olive (Olea europaea L.) DOP Umbria cultivars. Sex. Plant Reprod. 19:151-161. Russell, S.D. 1992. Double fertilization. Intl. Rev. Cytol. 140:357388.

Russell, S.D., M. Rourgier, and C. Dumas. 1990. Organization of the early post-fertilization megagametophyte of Populus deltoids ultrastructure and implications for male cytoplasmic transmission. Protoplasma 155:153-165.

Sethi, S.B. 1965. Structure and development of seed in Camellia sinensis (L.) O. Kuntze. Proc. Natl. Inst. Sci. India 31:25-33.

Snider, J.L., D.M. Oosterhuis, and E.M. Kawakami. 2011. Diurnal pollen tube growth rate is slowed by high temperature in field-grown Gossypium hirsutum pistils. J. Plant Physiol. 168:441-448.

Tan, X.F., D.Y. Yuan, J. Yuan, F. Zou, P. Xie, Y. Su, D.T. Yang, and J.T. Peng. 2011. An elite variety: Camellia oleifera 'Huashuo'. Scientia Silvae Sinicae 47:184-185 [in Chinese with English abstract].

Tsou, C. 1997. Embryology of the Theaceae-Anther and ovule development of Camellia, Franklinia, and Schima. Amer. J. Bot. 84:369.

Wachira, F.N. and S.K. Kamunya. 2005. Pseudo-self-incompatibility in some tea clones [Camellia sinensis (L.) O. Kuntze]. J. Hort. Sci. Biotechnol. 80:716-720.

Wang, X.N., Y.Z. Chen, S.F. Peng, and X.H. Yang. 2008. Five elite varieties of Camellia oleifera. Scientia Silvae Sinicae 44:173-174 [in Chinese with English abstract].

Weterings, K. and S.D. Russell. 2004. Experimental analysis of the fertilization process. Plant Cell 16:107-118.

Yu, H.S., B.Q. Huang, and S.D. Russell. 1994. Transmission of male cytoplasm during fertilization in Nicotiana tabacum. Sex. Plant Reprod. 7:313-323.

Zhang, D., L. Wang, and L.H. Zhuo. 2011. Embryology of Iris mandshurica Maxim. (Iridaceae) and its systematic relationships. Plant Syst. Evol. 293:43-52.

Zou, F., D.Y. Yuan, J.H. Duan, X.F. Tan, and L. Zhang. 2013a. A study of microsporgenesis and male gametogenesis in Camellia grijsii Hamce. Adv. J. Food Sci. Technol. 5:1590-1595.

Zou, F., D.Y. Yuan, X.F. Tan, P. Xie, T. Liao, X.M. Fan, and L. Zhang. 2013b. Megasporogensis and female gametophyte development of Camellia grijsii Hance. J. Chem. Pharmaceutical Res. 5: 484-488. 